\title{
ФОРМИРОВАНИЕ КРЕАТИВНОГО МЫШЛЕНИЯ ОБУЧАЕМЫХ ЧЕРЕЗ МАТЕМАТИЧЕСКИЕ КОЛЛОКВИУМЫ
}

\section{FORMATION OF STUDENTS' CREATIVE THINKING THROUGH MATHEMATICAL COLLOQUIUMS}

\section{Avetisyan \\ I. Shaposhnikova}

Summary: This article discusses the method of performing complex tasks on geometric constructions aimed at developing the research skills and creativity of trainees. The effectiveness of the mathematical colloquium on the development of creative qualities of students - flexibility, originality of thinking, etc. is noted. It is shown how it is possible within the framework of the colloquium to perform geometric constructions with the help of a compass and ruler and to perform sections of a polyhedron using information and communication technologies for the development of research skills of trainees.

Keywords: mathematical colloquium, research skills, creativity, construction tasks, polyhedron, polyhedron cross section.

\author{
Аветисян Миша Грантович \\ к.ф.-м.н., дочент, Сургутский государственный \\ университет \\ avetisjan56@gmail.com \\ Шапошникова Ирина Вадимовна \\ к.т.н., дочент, Сургутский государственный \\ университет \\ i-v-sh@mail.ru
}

Аннотация: В данной статье рассматривается методика выполнения сложных заданий на геометрические построения, нацеленная на развитие исследовательских навыков и креативности обучаемых. Отмечена эффективность математического коллоквиума на развитие креативных качеств обучаемых - гибкости, оригинальности мышления и др. Показано как возможно в рамках коллоквиума выполнять геометрические построения с помощью циркуля и линейки и выполнять сечения многогранника с использованием информационных и коммуникационных технологий для развития исследовательских умений обучаемых.

Ключевые слова: математический коллоквиум, исследовательские умения, креативность, задачи на построение, многогранник, сечение многогранника.

собностей учащихся при изучении математики, используются многие методические приемы. Коллоквиумы применяются при обучении в школах и особенно в вузах достаточно часто. В переводе с латинского colloquium «собеседование» - это вид учебных занятий, представляющих собой обсуждение под руководством преподавателя учебных тем. Обучаемый имеет уникальную возможность высказать свое мнение, точку зрения, обсудить возникшие вопросы с преподавателем. При собеседовании у учащегося имеется возможность проявить навыки аргументированно высказывать собственное мнение с помощью доказательств.

В качестве примера, рассмотрим подготовку заданий и проведение коллоквиумов в школе им. А.Н. Колмогорова (СУНЦ МГУ), нацеленных на развитие креативности школьников [2,3,5]. Математический коллоквиум включает в себя две функции: обучающую и контролирующую, таким образом проведение коллоквиума позволяет соединить процесс обучения и контроля.

Для проведения коллоквиума составляются задания по каждой изучаемой теме, часть из которых разбирается на уроках, часть предлагается для самостоятельной работы и изучения учащегося. Все учащиеся без исключения должны выполнить весь объем заданий, с подробными записями решений задач и доказательствами теорем. При этом обязательным требованием является

Для диагностики уровня развития творческих спо- 
общие правила оформления задания и его решения: четкие чертежи, полнота аргументации в решении задач, ясные ссылки на теоремы и ранее решенные задачи. Завершающим этапом коллоквиума является устная беседа учащегося с преподавателем по решенным и нерешенным задачам. Поскольку на данном этапе происходит персональный отчет учащихся по всем выполненным заданиям и для собеседования требуется время, рациональнее, если есть возможность, коллоквиумы проводить после уроков или выделять необходимое количество занятий во время обязательных часов.

Система математических коллоквиумов помогает четче организовать изучение той или иной темы и контроль за ее усвоением учащимися. При разработке коллоквиума следует придерживаться следующих методических принципов:

- выбирается только одна тема или какая-то ее часть;

- формируемый список заданий включает несколько типов задач - вычислительные, задачи на доказательство, задачи исследовательского характера;

- по возможности, включаются задания, связанные с историей развития математики;

- большинство задач объединены в логические блоки, каждый из которых включает базовые задачи.

При составлении заданий важное место уделяется эстетике математики (красивые рисунки, доказательства и пр.). Необходимо подбирать задания соответствующие специализации и направлению обучения учащегося, что будет способствовать развитию креативности и мотивации обучаемых к изучению данной математической темы. Методически правильнее решение трудных задач разбить на цепочку решений более простых задач. Исследовательские задачи должны иметь прикладной характер и должны быть нацелены на более глубокое изучение темы коллоквиума и на развитие мотивации к творческой деятельности, развитию креативности и интеллекта. Необходимо уделять внимание интеграционным связям различных областей математики для реализации межпредметных связей [3-7].

Система коллоквиумов достаточно эффективна, поскольку дает возможность учащемуся самостоятельно, дома, не торопясь выполнять учебные задания, оформлять решения в рабочей тетради с тщательно оформленными записями, что дисциплинирует его и мотивирует. Указанная система позволяет организовать деятельность учащегося таким образом, что в процессе работы он учится самостоятельно ставить перед собой задачи, искать методы их решения и делать вывод. Устная беседа с преподавателем дает возможность учащемуся защитить свою точку зрения, аргументировать от- вет, что неоспоримо в плане развития его творческих способностей и креативности мышления.

В качестве примера, рассмотрим тему для математического коллоквиума: «Задачи на геометрические построения». Геометрические построения являются особо значимыми элементами при изучении геометрии. Геометрические задачи на построение каких-либо объектов относятся к повышенному уровню сложности, их особенность заключается в том, что не существует единого алгоритма для решения всех таких задач. Каждое из подобных заданий по-своему уникально и требует индивидуального подхода для его решения. Подобные задания представляют собой материал для индивидуального поиска путей решений, т. е. для развития творческого мышления и позволяют использовать их для оценки креативных способностей учащихся.

Следует отметить следующие методические особенности проведения математического коллоквиума по теме «Задачи на геометрические построения»:

- задачи на геометрические построение остаются актуальной и важной темой в процессе изучения геометрии, поскольку компьютерные технологии с применением графических редакторов делают эти построения более наглядными, красивыми и понятными, что способно вызвать мотивацию у учащихся к изучению геометрии.

- задачи на геометрическое построение развивают геометрическое и объемное мышление гораздо полнее и острее, чем решение задач на вычисление, и способно вызвать увлечение работой, которое приводит к усилению любознательности и к желанию расширить и углубить изучение геометрии.

- основная цель решения любой задачи на построение состоит в том, чтобы построить некоторую фигуру, если даны соотношения между ее элементами. Решить задачу - это значит указать конечную последовательность построений, после выполнения которых, искомая фигура уже будет считаться построенной в силу аксиом геометрии. Кроме того, решить задачу на построение - значит найти последовательность всех шагов для её решения. Чтобы найти последовательность шагов, т. е. составить план построения, делают примерный чертёж искомой фигуры, отмечают те отрезки и углы, которые известны из условия задачи, стараются определить нахождение каждой искомой точки (прямой, угла) или величины. После этого стремятся найти такую зависимость между данными и искомыми величинами, которая позволяет найти (построить) искомую точку (прямую, угол), и составляют план построения. Составление плана - самая важная и сложная часть задачи, позволяющая раскрыть аналитические способности 
учащихся: умение анализировать, исследовать и креативно мыслить.

- выполнив анализ, наметив план, учащиеся описывают само построение. Оно должно содержать лишь основные построения и элементарные действия с линейкой и циркулем - это и гарантирует возможность построения. На этом этапе решения задачи учащиеся демонстрируют свои умения правильно и красиво выполнять геометрические построения.

Таким образом, математический коллоквиум по теме «Задачи на геометрические построения» содержит все этапы исследовательской деятельности:

1. постановка задачи;

2. предполагаемое решение задачи - догадка, гипотеза;

3. исследование догадки, гипотезы путем наблюдения, опыта, теоретического анализа;

4. разрешение задачи и проверка;

5. фиксирование результатов исследования в форме записи, рисунка.

Приведем несколько примеров решения задач на построение:

Задача 1. Построить прямоугольный треугольник по катету и сумме второго катета и гипотенузы.

\section{Дано:}

\section{Треугольник: $A B C$}

Kamem

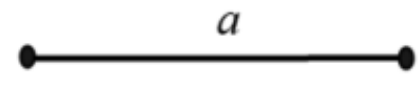

\section{Катет +гипотенуза:}
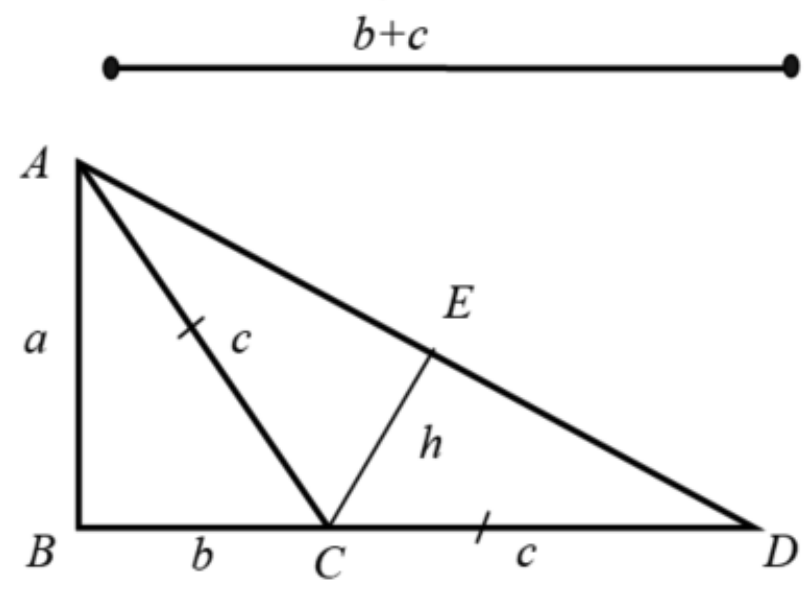

Рис. 1. Построение прямоугольного треугольника

Анализ решения:

Допустим, треугольник АВС (рис.1) прямоугольный и уже построен. Отложим на луче $\mathrm{BC}$ отрезок $\mathrm{CD}=$ с и соединим точки A и D. Достроенный треугольник ABD является также прямоугольным с катетами, $a$ и $(b+c)$. Построив его, получим вершины А и В искомого треугольника. Точка $C$ равноудалена от вершин $A$ и $D$, значит лежит на серединном перпендикуляре к $\mathrm{AD}$, т. е. $\mathrm{C}=\mathrm{EC} \cap \mathrm{BD}$, где $\mathrm{EC}$ - серединный перпендикуляр к AD.

Задача 2. Построить треугольник по медиане и двум углам, на которые она делит угол треугольника.

\section{Дано:}

медиана

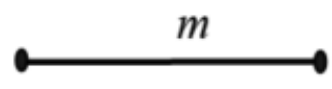

Угол $\alpha$ :
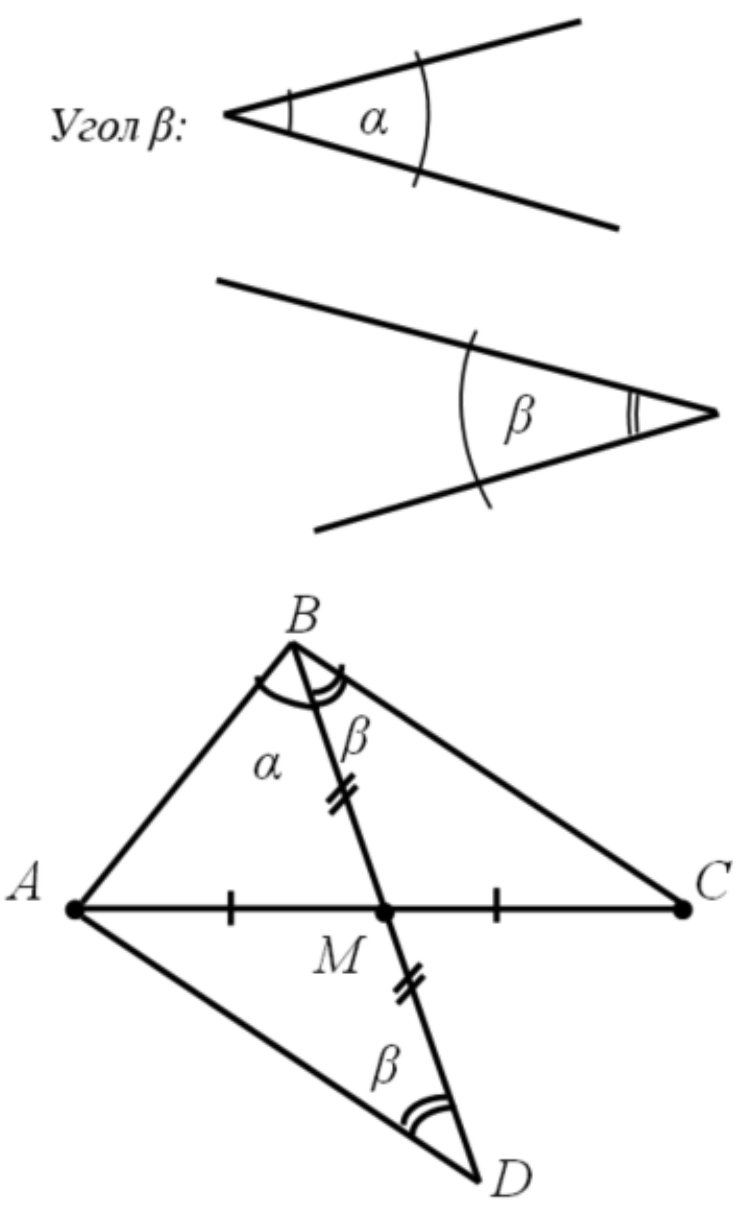

Рис. 2. Построение треугольника по медиане и двум углам

Анализ решения:

Допустим треугольник ABC построен (рис.2). Продолжим отрезок $\mathrm{BM}$ так, что $\mathrm{MD}=\mathrm{BM}=m$. Соединим точки $\mathrm{D}$ и $\mathrm{A} . \triangle \mathrm{AMD}=\triangle \mathrm{CMB}$ по двум сторонам и углу между ними. Тогда $\angle A D M=\angle C B M=\beta$. Получим вспомогательный треу- 
гольник $A B D$, который может быть построен по стороне $2 m$ и двум прилежащим углам а и $\beta$. Осталось найти вершину C: $C=A M \cap D R$, где $M$ - середина BD, DR - сторона $\angle \mathrm{DBR}=\beta$

Задача 3. Построить квадрат по разности диагонали $d$ и стороны $a$.

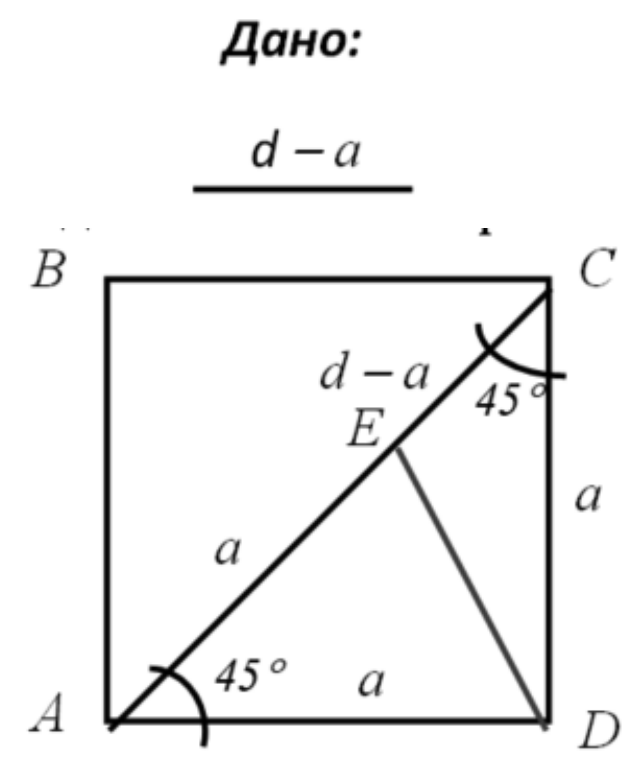

Рис. 3. Построение квадрата

Анализ решения:

Допустим квадрат ABCD построен (рис. 3). На диагонали $\mathrm{AC}$ от точки $\mathrm{C}$ отложим отрезок $\mathrm{CE}=d-a$. Угол $\angle D A E$ равен $45^{\circ}$, треугольник AED - равносторонний, значит $\angle \mathrm{AED}=\frac{180^{\circ}-45^{\circ}}{2}=\frac{135^{\circ}}{2}$, а следовательно, $\angle \mathrm{CED}=180-\frac{135^{\circ}}{2}=90^{\circ}+\frac{45^{\circ}}{2}$. Вспомогательный треугольник CED может быть построен по стороне и двум прилежащим углам. Останется достроить до квадрата.

При решении подобных задач на построение с помощью циркуля и линейки обучаемому, с одной стороны, необходимо использование исходного образа (треугольника, квадрата и др.), с другой - максимальное отвлечение от этого образа при дополнительных построениях, что способствует развитию интуитивного мышления важнейшего креативного качества. После того, как обучаемый произведет мысленное преобразование образа, ему необходимо построить данную фигуру на доске или листе бумаги.

В качестве примера, рассмотрим еще одну тему коллоквиума «Построение сечений многогранников» и отметим некоторые методические замечания:

- построение сечений является очень важной темой в процессе изучения пространственной геометрии: сечения позволяют лучше развить пространственное мышление и, кроме того, их использование является одним из общих методов решения стереометрических задач;

- при построении сечений требуется проявить знания и стереометрии, и планиметрии, поэтому задачи на построение сечений связывают эти два раздела геометрии и одной из целей данного коллоквиума является формирование навыков выявлять эти связи, то есть умения строить сечения;

- особенностью коллоквиума является наличие большого количества красивых чертежей, которые способствуют увеличению интереса учащихся к предмету;

- задание коллоквиума предшествует ряду заданий математического практикума: «Построение сечений икосаэдра, заданного тремя точками», «Построение центральной проекции на плоскость фигуры, составленной из кубиков», «Построение центральной проекции правильного плоского паркета на другую плоскость» и др.;

- коллоквиум является важным инструментом для подготовки учащихся к осознанному восприятию идей проективной геометрии.

Применение метода сечений для решения различных задач стереометрии и формулируют десятки вопросов, ответ на которые требует от обучающегося творческого подхода и упорной работы, что позитивно влияет на развитие креативности обучаемых.

- примерами теоретических вопросов, исследуемых в процессе проведения данного коллоквиума, могут служить следующие задания:

а) что такое сечение многогранника плоскостью и как оно задается;

б) какие плоские фигуры могут получиться при сечении параллелепипеда плоскостью;

в) какие правильные многоугольники можно получить при пересечении куба плоскостью и др.

В теме «Сечение многогранников» авторами рассмотрено множество интересных и трудных задач. Выполнение коллоквиума нацелено на развитие таких креативных качеств как гибкость, оригинальность мышления, преодоление стереотипов мышления.

Для развития креативных качеств и мотивации к информатике и математике было бы полезно использовать информационные и коммуникационные технологии. Например, при изучении темы - сечение многогранников, используется PowerPoint при решении геометрической задачи: четырехугольная призма $A B C D A_{1} B_{1} C_{1} D_{1}$ пересечена плоскостью, проходящей через точки $\mathrm{M}, \mathrm{N}$ и $\mathrm{P}$, лежащих на боковых ребрах призмы. Построить сечение.

При построении данного сечения обучаемому с одной стороны необходимо использование исходного об- 
раза (точки М, N, P) с другой - максимальное отвлечение от этого образа при дополнительных построениях, что так же способствует развитию креативного мышления. После проделанных операций обучаемому целесообразно проанализировать слайды (см. пошаговое построение сечения на рисунках, расположенных ниже).

При запуске программы PowerPoint в режиме "Анимация" данные слайды будут восприниматься обучаемым, как неразрывные составляющие воспроизводимого на мониторе сечения.

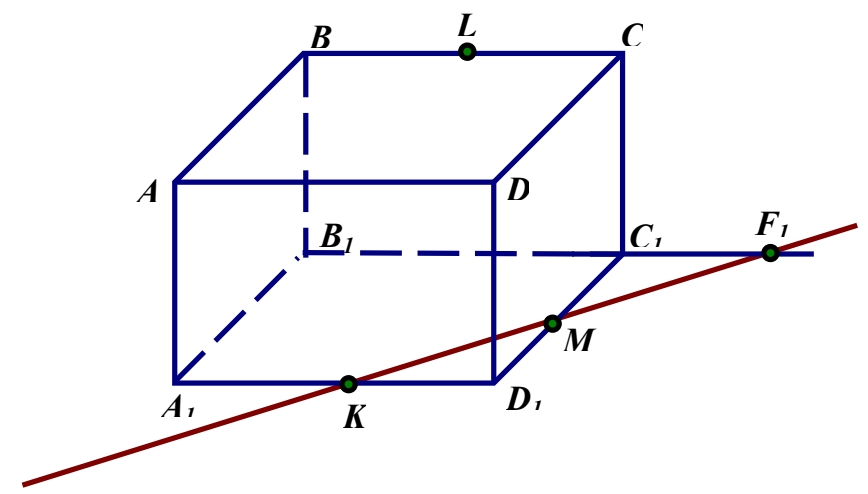

Рис. 4. Первый шаг построения сечения

- Первый шаг построения плоскости (рис. 4):

Проводим прямую, проходящую через точки К и М.

Рисуем продолжение ребра $\left(\mathrm{B}_{1} \mathrm{C}_{1}\right)$. Точка пересечения этих прямых - F1.

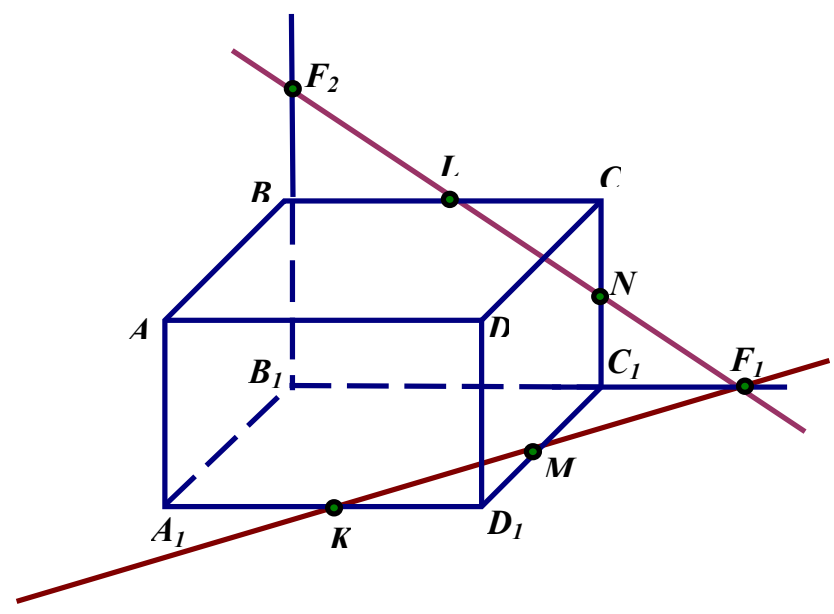

Рис. 5. Второй шаг построения сечения

- $\quad$ Второй шаг построения сечения (рис. 5):
Проводим прямую, проходящую через точки $\mathrm{F}_{1}$ и L.

Пересечения прямой $\left(\mathrm{F}_{1}, \mathrm{~L}\right)$ с ребром $\left(\mathrm{CC}_{1}\right)$ - точка $\mathrm{N}$.

Рисуем продолжение ребра (B, B) вверх.

Пересечение прямой $\left(F_{1}, L\right)$ и $\left(B_{1} B\right)$ - точка $F_{2}$.

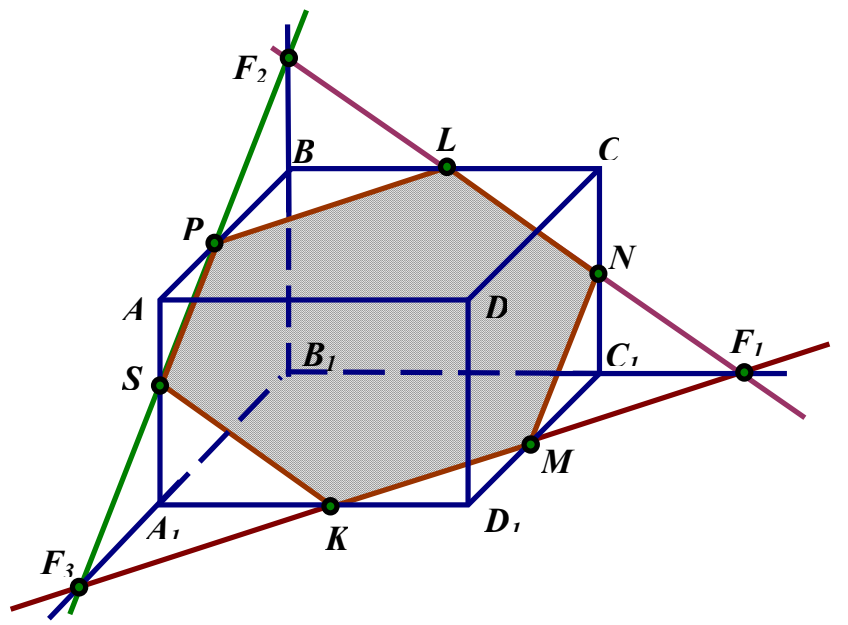

Рис. 6. Третий шаг построения сечения

- $\quad$ Третий шаг построения сечения (рис. 6):

Рисуем продолжение ребра $\left(\mathrm{B}_{1} \mathrm{~A}_{1}\right)$ до пересечения c прямой $(\mathrm{K}, \mathrm{M})$ - точка $\mathrm{F}_{3}$.

Проводим прямую через точки $\mathrm{F}_{2}$ и $\mathrm{F}_{3}$.

Ищем точку пересечения прямой $\left(\mathrm{F}_{2^{\prime}} \mathrm{F}_{3}\right)$ и ребра $(A, B)-P$.

Точка пересечения прямой $\left(F_{2}, F_{3}\right)$ и ребра $\left(A, A_{1}\right)-S$.

Соединяем точки S, P, L, N, M, K - получаем искомую плоскость.

\section{BывOА}

Целью математического образования должно быть развитие навыков оперирования с числами и фигурами, пространственного воображения, логического мышления. При решении математических задач наблюдается и развитие креативности обучаемых. Обладание интуицией, гибким и критичным мышлением дает возможность школьнику и студенту решать широкий круг задач. Выполнение обучаемыми математических коллоквиумов позитивно влияет на развитие креативности.

\section{ЛИТЕРАТУРА}

1. Вавилов В.В. Школа математического творчества. - М.: РОХОС, 2004. - 72 с.

2. Вавилов В.В., Красников П.М. Математические коллоквиумы. Часть1. - М.: Школа им. А.Н. Колмогорова СУНЦ МГУ. - 2006. - 64 с. 
3. Вавилов В.В., Красников П.М. Математические коллоквиумы. Часть2. - М.: Школа им. А.Н. Колмогорова СУНЦ МГУ. - 2006. - 36 с.

4. Глазкова К.Р. Уроки-исследования: формирование творческой, критически мыслящей личности: методическое пособие / К.Р. Глазкова, С.А. Живодробова // Математика. - М.: Первое сентября. - 2019. - №24. - С. 29-31

5. Крылов А.М. Учебно-исследовательская деятельность учащихся в процессе изучения математики: методическое пособие / А.М. Крылов. - М.: Омск. 2019. -73 C.

6. Савенков А.И. Содержание и организация исследовательского обучения школьников // Библиотека журнала «Директор школы». - 2003. - №8. - 204с.

7. Секованова Л.А., Шапошникова И.В. Разветвляющиеся и циклические программы. Табулирование функции (учебно-методические указания). - Кострома, КТИ, РИС, 1994. -24 с.

(с Аветисян Миша Грантович (avetisjan56@gmail.com), Шапошникова Ирина Вадимовна (i-v-sh@mail.ru)

Журнал «Современная наука: актуальные проблемы теории и практики»

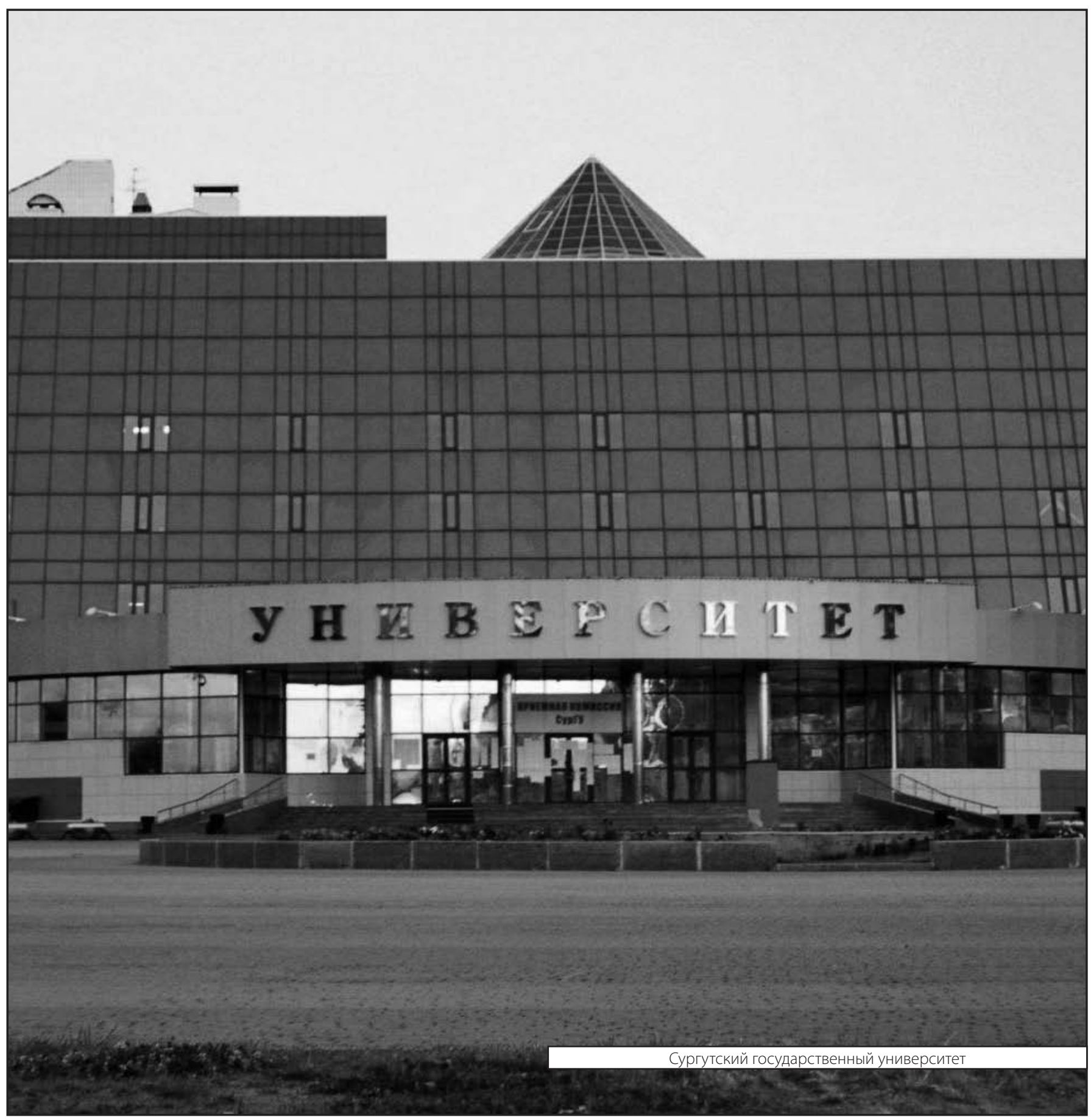

